\title{
Macrodeformation Twins in Single-Crystal Aluminum
}

\author{
F. Zhao, ${ }^{1,2}$ L. Wang, ${ }^{1}$ D. Fan, ${ }^{1}$ B. X. Bie, ${ }^{1,3}$ X. M. Zhou, ${ }^{1}$ T. Suo,${ }^{4}$ Y. L. Li,${ }^{4}$ M. W. Chen,,${ }^{5,6}$ \\ C. L. Liu, ${ }^{7}$ M. L. Qi, ${ }^{3}$ M. H. Zhu, ${ }^{2, *}$ and S. N. Luo ${ }^{1,2, \dagger}$ \\ ${ }^{1}$ The Peac Institute of Multiscale Sciences, Chengdu, Sichuan 610031, People's Republic of China \\ ${ }^{2}$ Key Laboratory of Advanced Technologies of Materials, Ministry of Education, \\ Southwest Jiaotong University, Chengdu, Sichuan 610031, People's Republic of China \\ ${ }^{3}$ School of Science, Wuhan Univiersity of Technology, Wuhan, Hubei 430070, People's Republic of China \\ ${ }^{4}$ School of Aeronautics, Northwestern Polytechnical University, Xi'an, Shaanxi 710072, \\ People's Republic of China \\ ${ }^{5}$ WPI Advanced Institute for Materials Research, Tohoku University, Sendai 980-8577, Japan \\ ${ }^{6}$ State Key Laboratory of Metal Matrix Composites, School of Materials Science and Engineering, \\ Shanghai Jiao Tong University, Shanghai 200030, People's Republic of China \\ ${ }^{7}$ Institute of Fluid Physics, Mianyang, Sichuan 621900, People's Republic of China
}

(Received 15 October 2015; published 17 February 2016)

\begin{abstract}
Deformation twinning in pure aluminum has been considered to be a unique property of nanostructured aluminum. A lingering mystery is whether deformation twinning occurs in coarse-grained or single-crystal aluminum at scales beyond nanotwins. Here, we present the first experimental demonstration of macrodeformation twins in single-crystal aluminum formed under an ultrahigh strain rate $\left(\sim 10^{6} \mathrm{~s}^{-1}\right)$ and large shear strain $(200 \%)$ via dynamic equal channel angular pressing. Large-scale molecular dynamics simulations suggest that the frustration of subsonic dislocation motion leads to transonic deformation twinning. Deformation twinning is rooted in the rate dependences of dislocation motion and twinning, which are coupled, complementary processes during severe plastic deformation under ultrahigh strain rates.
\end{abstract}

DOI: 10.1103/PhysRevLett.116.075501

When we talk about crystal deformation, what do we actually talk about? Crystal defects [1]. Crystal defects such as dislocations (line defects) and twins (planar defects) play a critical role in plastic deformation and ultimately govern multifarious mechanical behaviors of many crystalline materials $[2,3]$. While both dislocation slip and deformation twinning are dependent on an intrinsic material propertystacking fault energy (SFE) [4,5] - their sensitivities to SFE differ considerably. A notable example is pure aluminum, a typical face-centered cubic (fcc) metal with high SFE (104-142 $\mathrm{mJ} \mathrm{m}^{-2}$ ) [6], in which deformation twinning rarely occurs-even deformed at low temperatures and/ or at high strain rates $[7,8]$. This rareness of deformation twinning in such materials is normally attributed to the following two reasons: (i) a large number of slip systems in fcc metals render dislocation slip a very efficient deformation mode $[9,10]$, and (ii) the nucleation of twinning partial dislocations require much higher shear stresses than trailing partial dislocations due to high unstable twin fault energy [11]. Searching for macrodeformation twins in pure aluminum and revealing underlying mechanisms have been of sustained interest in the past decades.

Molecular dynamics (MD) simulations first predicted that nanoscale deformation twins can nucleate under high tensile stress $(2.5 \mathrm{GPa})$ and high strain rate $\left(10^{7} \mathrm{~s}^{-1}\right)$ in nanograined aluminum $[6,12]$, and subsequent experiments confirmed this prediction in nanograined aluminum films under different kinds of severe plastic deformation [13-15]. One explanation was proposed based on classical dislocation theory [16]: when grain size decreases to tens of nanometers, normal dislocation activities are greatly suppressed by the high fraction of grain boundaries; as a result, deformation twinning takes over as the dominant deformation mechanism [13]. Besides nanograin size effect, many simulation and experimental studies suggest that deformation twinning prefers to occur at high strain rates [17-19] and large shear strain [19] in fcc metals. This rate-dependent twinning mechanism was corroborated by a very recent experiment on pure aluminum with comparatively large nanograins (50-100 nm) [20]. However, there has been no solid evidence for deformation twinning in single-crystal or coarse-grained pure aluminum. It is natural to ask whether deformation twinning indeed occurs in coarse-grained and single-crystal aluminum, and if it does, whether the mechanisms are the same as those in nanostructured aluminum. In other words, is deformation twinning simply unique to nanostructured aluminum?

In the present work, we develop a novel dynamic equal channel angular pressing (D-ECAP) technique, schematically illustrated in Fig. 1(a) and Fig. S1 of the Supplemental Material [21], to enable large shear plastic deformation at an ultrahigh strain rate to investigate twinning in single-crystal aluminum. In the traditional ECAP method, the piston is pressed at a low speed $\left(10^{-3} \mathrm{~m} \mathrm{~s}^{-1}\right)$, while in D-ECAP a much higher piston velocity $\left(10^{2} \mathrm{~m} \mathrm{~s}^{-1}\right)$ is applied to induce ultrahigh strain rates $\left(10^{6} \mathrm{~s}^{-1}\right)$. 
(a)

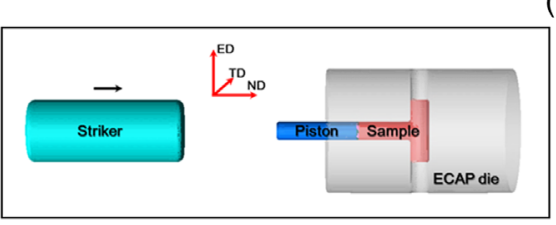

(c)

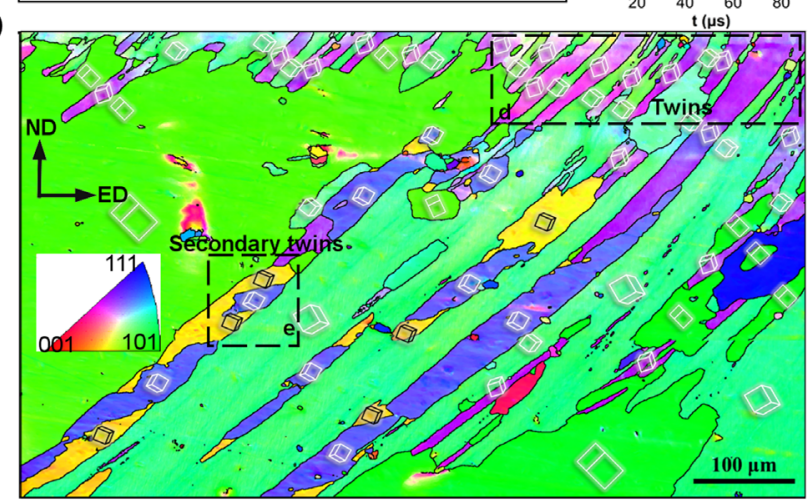

(d)

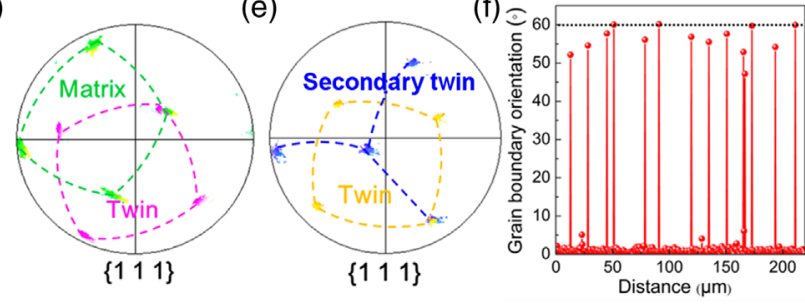

FIG. 1. Dynamic equal channel angular pressing (D-ECAP) experiments and a microstructure overview of macrodeformation twins in single-crystal aluminum. (a) Schematic of the D-ECAP setup. (b) Evolution of plastic strain in the specimen under D-ECAP and the estimated strain rate (the dashed line). (c) Typical inverse pole figure (IPF) map of high-density macrodeformation twins. (d),(e) Pole figures of deformation twins and secondary twins corresponding to regions (d) and (e) marked in (c). (f) Twin boundary misorientation profile in region (d).

A single-crystal aluminum rod [ED-[010], TD-[101], ND-[101], Figs. 1(a) and 1(c)] with a purity of $99.999 \%$, $3 \mathrm{~mm}$ in diameter, is set in the D-ECAP die, then extruded for only one pass by a high velocity pressing at room temperature. The estimated maximum strain rate from finite elemental analysis is about $10^{6} \mathrm{~s}^{-1}$ (see Fig. S2 of the Supplemental Material [21]) and the corresponding plastic strain is about 200\% [see Fig. 1(b)]. After extrusion via D-ECAP, the specimen is characterized by electron backscattering diffraction (EBSD). High-density macrodeformation twins are observed near the upper extrusion surface [see Fig. 1(c) and Figs. S3 and S4 of the Supplemental Material [21]). Such macrodeformation twins had never been found previously in pure aluminum, either in nanocrystalline, ultrafine, coarse-grained, or single-crystal aluminum.

An example of EBSD analysis of the recovered specimen after D-ECAP is shown in an inverse pole figure (IPF) map [see Fig. 1(c)]. The average twin width is about $40 \mu \mathrm{m}$ and the maximum length is about $500 \mu \mathrm{m}$. The twin boundaries are determined to be typical $\Sigma 3\{111\} 60^{\circ}$ coherent twin boundaries (see Fig. S3 of the Supplemental Material [21]). Figure 1(c) also shows that all of the macrodeformation twins nucleate from the upper surface where shear deformation is augmented by the sample-die friction and propagate toward the sample interior along the shear direction $([\overline{1} \overline{2} \overline{1}])$. However, unlike normal straight twin boundaries, the deformation twin boundaries induced by the D-ECAP process are curved, indicating rotation. This is caused by strong interactions between twins and dislocations under severe shear plastic deformation. Numerous low-angle grain boundaries (LAGBs), which correspond to high-density dislocation structures, are found in the vicinity of twin boundaries (see Figs. S5 and S6 of the Supplemental Material [21]). Occasionally, secondary deformation twins form at preceding twin boundaries [see Fig. 1(c)]. These primary and secondary deformation twins are identified from pole figure maps, and the twin planes are $\{111\}$ [see Figs. 1(d) and 1(e)]. Grain boundary orientation analysis reveals that the average misorientations are approximately $60^{\circ}$, and the slight deviations from this angle are caused by twin boundary rotation [see Fig. 1(f)].

The deformation twins in single-crystal aluminum processed with D-ECAP are "macroscopic" in nature, distinct from the nanotwins observed previously in nanograined aluminum [13-15]. In nanograined aluminum, as grain size decreases to below a critical value $(\sim 15 \mathrm{~nm})$, the critical stress for dislocation nucleation becomes higher than that for deformation twinning and, therefore, deformation twinning replaces dislocation slip as the preferred deformation mode [13]. However, nanoscale grain boundaries can also strongly restrict twin propagation, thus limiting twin size to several nanometers. Our experiments demonstrate that deformation twins can be induced by simultaneous ultrahigh strain rates (via dynamic loading) and large shear deformation (via ECAP), and grow to submillimeter scales in single-crystal aluminum without grain boundaries serving as the barrier to twin propagation. MD simulations suggest that the spontaneous self-pinning of dislocations impedes dislocation motion at high strain rates, and thus deformation twinning takes over as the predominant deformation mode. Our experimental results confirm this prediction [43].

Figure 2(a) shows abundant deformation twins with a typical lens shape formed within the sample interior, which has undergone less friction and smaller shear strain. During severe plastic deformation, high-density dislocation structures (LAGBs) are found to cluster around twin boundaries, suggesting that deformation twins can serve as dislocation sources as well as barriers, effectively hindering dislocation motion around them [see Figs. 2(b) and 2(c)]. To further verify this observation, twin and grain boundary fractions are quantified from the IPF map. As shown in Fig. 2(d), LAGBs, or high-density dislocation structures, are the most prominent microstructure feature. During such ultrahigh 
(a)

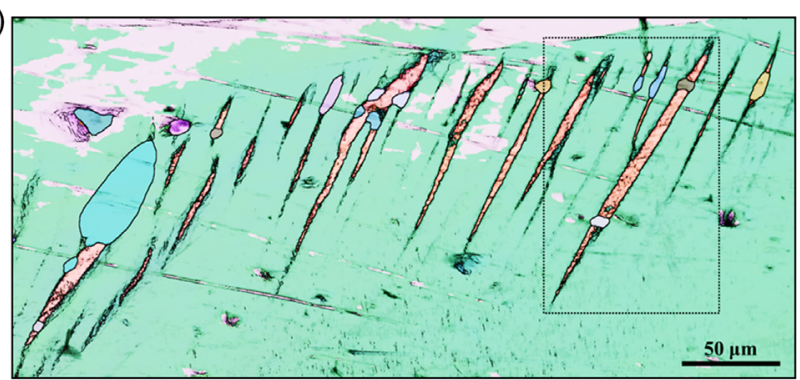

(b)

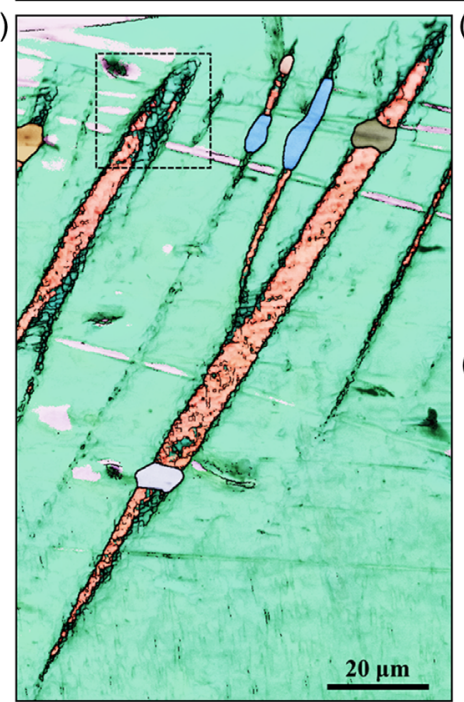

(a)

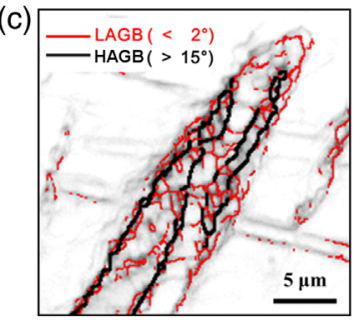

(d)

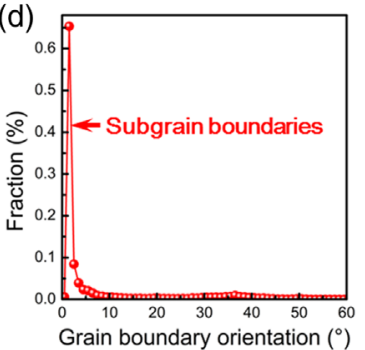

(c)

FIG. 2. Deformation twin boundaries, low-angle grain boundaries (LAGBs), and high-angle grain boundaries (HAGBs) in an aluminum specimen after D-ECAP. (a) EBSD image of deformation twins formed in the interior of the specimen. (b) Distribution of LAGBs (the black lines) around deformation twins. (c) Magnified image of the region delimited by the rectangle in (b) highlighting interactions between twin boundaries and LAGBs. Twin boundaries can act as dislocation sources and barriers to dislocation motion. (d) Misorientation distribution corresponding to (b), indicating that LAGBs or high-density dislocation structures are the major deformation microstructure within deformation twins.

strain-rate shear deformation, dislocation nucleation and interactions are still significant near and within deformation twin boundaries, and the propagation of deformation twins assists dislocation nucleation as new dislocation sources (see Fig. S5 of the Supplemental Material [21]). Our experiments provide direct evidence that dislocation slip and deformation twinning can act together as complementary deformation modes during ultrahigh strain-rate shear deformation. While the transition from dislocation slip to twinning occurs in aluminum only when dislocation activities are stagnant, dislocation nucleation is actually enhanced during twin propagation, in contrast to previous views.

In order to help reveal phenomena and underlying mechanisms related to deformation twinning under ultrahigh strain-rate shear deformation within the context of D-ECAP experiments, MD simulations are carried out. The simulation details are presented in the Supplemental Material [21]. In
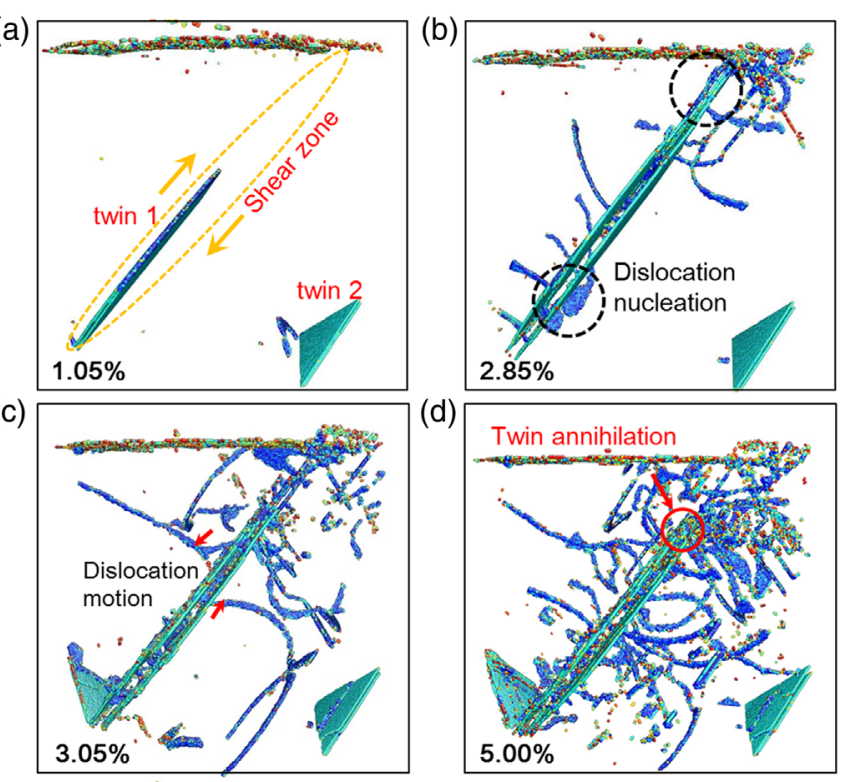

(d)

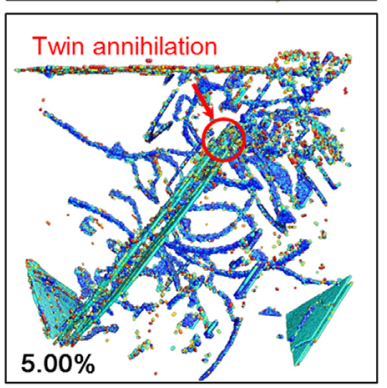

FIG. 3. Snapshots of deformation twinning and interactions between twin boundaries and dislocations, obtained from MD simulations. A movie of the simulations is included in the Supplemental Material [21]. (a) Deformation twin nucleation and propagation in the shear zone. (b) Dislocation nucleation from twin boundaries, as indicated by the circles. (c) Dislocation motion around twin boundaries. (d) Twin annihilation caused by the interactions between twin boundaries and dislocations.

our MD simulations mimicking the D-ECAP experiments, the pressing velocity is relatively low $\left(10 \mathrm{~m} \mathrm{~s}^{-1}\right)$, but the corresponding strain rate is still high $\left(\sim 10^{8} \mathrm{~s}^{-1}\right)$ given the relatively small length scales. When the sample initially passes through the die corner, the leading partial dislocations first emit from this area [see Fig. 3(a)]. The resolved shear stress is about $1 \mathrm{GPa}(1.05 \%$; see Fig. S8 of the Supplemental Material [21]), comparable to twin nucleation stress reported previously, 0.64 GPa [13] and $0.93 \mathrm{GPa}$ $[44,45]$. Consequently, the twin partial dislocations immediately appear following the leading partial dislocations, and a nanotwin then forms. Figures 3(b) and 3(c) show that, as deformation twins propagate, dislocations nucleate from the twin boundaries, and this greatly enhances the local deformation ability in order to accommodate the high deformation rate. On the other hand, dislocations quickly become selfpinned and tangled (see Fig. S9 of the Supplemental Material [21]) as they move away from twin planes. High-density dislocation structures (i.e., LAGBs) arise near the twin boundaries, consistent with our experimental results. When the propagation of deformation twins ceases, dislocation motion and dislocation interactions begin to dominate plastic deformation, and annihilation of certain twins occurs as a result [see Fig. 3(d)].

It has been shown that deformation twins propagate faster at a high loading rate in a number of materials $[9,46,47]$. Our D-ECAP experiments and MD simulations demonstrate the coexistence of deformation twinning and 


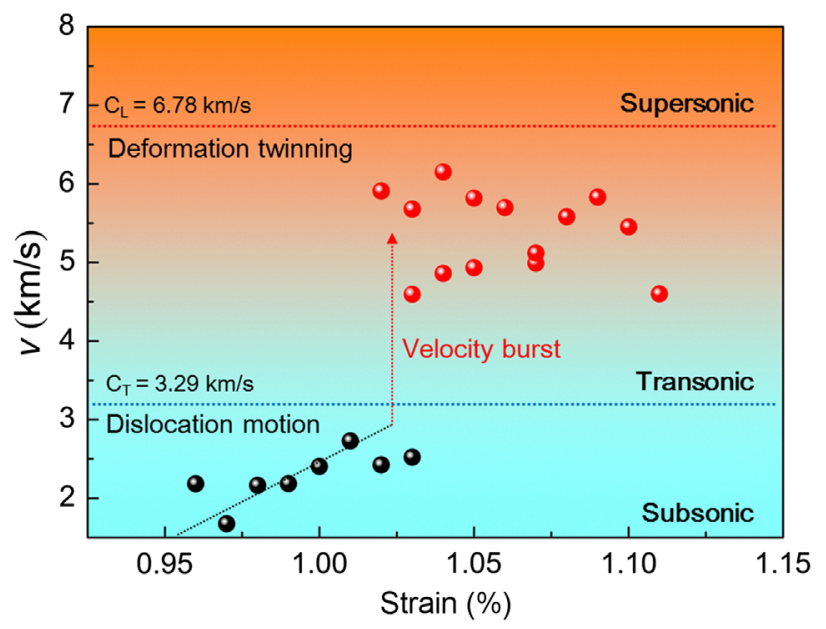

FIG. 4. Propagation speed of dislocations and deformation twins as a function of volumetric strain during D-ECAP obtained from MD simulations.

dislocation slip in single-crystal aluminum. Therefore, it is desirable to compare the propagation speeds of dislocation motion and deformation twinning during high-rate shear deformation. Figure 4 shows that the leading partial dislocations nucleate first, and the speed of dislocation motion increases with increasing strain but remains subsonic. Subsequent transition to deformation twinning leads to a velocity burst, and deformation twins propagate at a transonic speed. Classical dislocation theory predicts that dislocation velocity cannot exceed the shear wave velocity [16] and, with increasing dislocation velocity, dislocation selfpinning and tangling ensue, leading to stress accumulation until deformation twins nucleate $[48,49]$. Twin propagation velocity exceeds the shear wave velocity. The enormous propagation velocity jump (subsonic to transonic) is a direct result of the dislocation slip-twinning transition in singlecrystal aluminum necessitated by the ultrahigh strain rate.

The D-ECAP experiments and MD simulations reveal that deformation twinning and dislocation slip coexist in single-crystal aluminum under ultrahigh strain-rate loading. The applied strain rate, $\dot{\varepsilon}_{\text {applied }}$, consists of contributions from dislocation motion and deformation twinning:

$$
\dot{\varepsilon}_{\text {applied }}=\dot{\varepsilon}_{\text {dislocation }}+\dot{\varepsilon}_{\text {twin }} .
$$

Based on classical dislocation theory [16], $\dot{\varepsilon}_{\text {dislocation }}=$ $m b \rho v$, where $m$ is a material-specific parameter, $b$ is the Burgers vector amplitude, $\rho$ is the mobile dislocation density, and $v$ is the average dislocation propagation velocity. Twin boundaries can serve as new dislocation sources while propagating and, subsequently, the mobile dislocation density increases rapidly due to fast twin propagation. Then Eq. (1) can be rewritten as

$$
\dot{\varepsilon}_{\text {applied }}=m b v\left(\rho_{0}+\rho_{\mathrm{twin}} N L v_{\mathrm{twin}} t^{2}\right)+\dot{\varepsilon}_{\mathrm{twin}},
$$

where $\rho_{0}$ is the initial mobile dislocation density, $\rho_{\text {twin }}$ is the twin density, $N$ is the dislocation nucleation rate, $v_{\text {twin }}$ is the propagation velocity of a deformation twin, and $L$ is the average width of the twins (see Fig. S10 of the Supplemental Material [21]).

If deformation involves dislocation motion only, then $\dot{\varepsilon}_{\text {dislocation }}=m b v \rho_{0}$ and is limited by $v$, which is subsonic. As a result, dislocation itself cannot accommodate the applied strain rate for a sufficiently high $\dot{\varepsilon}_{\text {applied, }}$, and the strain deficit has to be supplemented by faster propagating deformation twinning (transonic twinning; see Fig. 4). Dislocation self-pinning and tangling slow down dislocation motion and give rise to intense stress accumulation and subsequent deformation twinning. New dislocations nucleate at propagating twin boundaries (as new dislocation sources) [50,51], and mobile dislocation density increases by $\rho_{\text {twin }} N L v_{\text {twin }} t^{2}$. Thus, dislocation frustration leads to deformation twinning, which in turn assists dislocation nucleation. In other words, dislocation slip and deformation twinning coexist and complement each other necessarily in high-rate severe plastic deformation of aluminum, as seen in our experiments and in MD simulations.

Our D-ECAP experiments and MD simulations have demonstrated that deformation twinning is not unique to nanostructured aluminum, and macrodeformation twinning can occur in single-crystal-and likely coarse-grainedaluminum under ultrahigh strain rates and large shear. Deformation twinning and dislocation slip are ratecontrolled, coupled, and complementary processes during ultrahigh strain-rate plastic deformation. Deformation twinning, once elusive in experiments, may indeed play a major role in plastic deformation under high strain rates for aluminum and, conceivably, other high-SFE metals.

The work was partially supported by the 973 Project of China (Grant No. 2014CB845904), and the NSF and the NSAF of China (Grants No. 11472253 and No. U1230202).

F. Z. and L. W. contributed equally to this work.

*zhuminhao@swjtu.cn

†sluo@pims.ac.cn

[1] J. R. Greer, Nat. Mater. 12, 689 (2013).

[2] J. W. Christian and S. Mahajan, Prog. Mater. Sci. 39, 1 (1995).

[3] Y. T. Zhu, X. Z. Liao, and X. L. Wu, Prog. Mater. Sci. 57, 1 (2012).

[4] H. Van Swygenhoven, P. M. Derlet, and A. G. Froseth, Nat. Mater. 3, 399 (2004).

[5] V.S. Sarma, J. Wang, W. W. Jian, A. Kauffmann, H. Conrad, J. Freudenberger, and Y. T. Zhu, Mater. Sci. Eng. A 527, 7624 (2010)

[6] V. Yamakov, D. Wolf, S. R. Phillpot, A. K. Mukherjee, and H. Gleiter, Nat. Mater. 1, 45 (2002).

[7] J. H. Jiang, Y. Ding, F. Q. Zuo, and A. D. Shan, Scr. Mater. 60, 905 (2009). 
[8] Q. Liu, D. J. Jensen, and N. Hansen, Acta Mater. 46, 5819 (1998).

[9] N. P. Daphalapurkar, J. W. Wilkerson, T. W. Wright, and K. T. Ramesh, Acta Mater. 68, 82 (2014).

[10] T. H. Blewitt, P. R. Coltman, and J. K. Redman, J. Appl. Phys. 28, 651 (1957).

[11] D. H. Warner, W. A. Curtin, and S. Qu, Nat. Mater. 6, 876 (2007).

[12] V. Yamakov, D. Wolf, S. R. Phillpot, and H. Gleiter, Acta Mater. 50, 5005 (2002).

[13] M. W. Chen, E. Ma, K. J. Hemker, H. W. Sheng, Y. M. Wang, and X. M. Cheng, Science 300, 1275 (2003).

[14] X. Z. Liao, F. Zhou, E. J. Lavernia, S. G. Srinivasan, M. I. Baskes, D. W. He, and Y. T. Zhu, Appl. Phys. Lett. 83, 632 (2003).

[15] X. Z. Liao, F. Zhou, E. J. Lavernia, D. W. He, and Y. T. Zhu, Appl. Phys. Lett. 83, 5062 (2003).

[16] J. P. Hirth and J. Lothe, Theory of Dislocations (Krieger Publishing, Malabar, FL, 1992).

[17] M. A. Meyers, O. Vohringer, and V. A. Lubarda, Acta Mater. 49, 4025 (2001).

[18] G. H. Xiao, N. R. Tao, and K. Lu, Scr. Mater. 59, 975 (2008).

[19] T. L. Brown, C. Saldana, T. G. Murthy, J. B. Mann, Y. Gao, A. H. King, W. Dale Compton, K. P. Trumble, and S. Chandrasekar, Acta Mater. 57, 5491 (2009).

[20] B. Cao, N. P. Daphalapurkar, and K. T. Ramesh, Meccanica 50, 561 (2015).

[21] See Supplemental Material at http://link.aps.org/ supplemental/10.1103/PhysRevLett.116.075501, which includes Refs. [22-42], for details on the experiments and simulations.

[22] R. Z. Valiev, Nat. Mater. 3, 511 (2004).

[23] R. Z. Valiev, R. K. Islamgaliev, and I. V. Alexandrov, Prog. Mater. Sci. 45, 103 (2000).

[24] Y. Iwahashi, Z. Horita, M. Nemoto, and T. G. Langdon, Acta Mater. 46, 3317 (1998).

[25] M. S. Duesbery, Model. Simul. Mater. Sci. Eng. 6, 35 (1998).

[26] T. Vegge, T. Leffers, O. B. Pedersen, and K. W. Jacobsen, Mater. Sci. Eng. A 319-321, 119 (2001).

[27] D. J. Bacon, U. F. Kocks, and R. O. Scattergood, Philos. Mag. 28, 1241 (1973).

[28] S. Plimpton, J. Comput. Phys. 117, 1 (1995); see also http:// lammps.sandia.gov.
[29] X. Y. Liu, F. Ercolessi, and J. B. Adams, Model. Simul. Mater. Sci. Eng. 12, 665 (2004).

[30] F. Ercolessi and J. B. Adams, Europhys. Lett. 26, 583 (1994).

[31] L. Wang, J. C. E, Y. Cai, F. Zhao, D. Fan, and S. N. Luo, J. Appl. Phys. 117, 084301 (2015).

[32] S. N. Luo, Q. An, T. C. Germann, and L. B. Han, J. Appl. Phys. 106, 013502 (2009).

[33] S. N. Luo, T. C. Germann, and D. L. Tonks, J. Appl. Phys. 107, 123507 (2010).

[34] Q. An, Y. Liu, S. V. Zybin, H. J. Kim, and W. A. Goddard III, J. Phys. Chem. C 116, 10198 (2012).

[35] Y. Cai, F. P. Zhao, Q. An, H. A. Wu, W. A. Goddard III, and S. N. Luo, J. Chem. Phys. 139, 164704 (2013).

[36] G. J. Ackland and R. Thetford, Philos. Mag. A 56, 15 (1987).

[37] J. Li, Model. Simul. Mater. Sci. Eng. 11, 173 (2003).

[38] P. Gumbsch and H. J. Gao, Science 283, 965 (1999).

[39] J. Weertman and J. R. Weertman, Elementary Dislocation Theory (Oxford University Press, New York, 1992).

[40] L. Lu, Y.F. Shen, X. H. Chen, L. H. Qian, and K. Lu, Science 304, 422 (2004).

[41] L. Lu, X. H. Chen, X. Huang, and K. Lu, Science 323, 607 (2009).

[42] X. Y. Li, Y. J. Wei, L. Lu, K. Lu, and H. J. Gao, Nature (London) 464, 877 (2010).

[43] J. Marian, W. Cai, and V. V. Bulatov, Nat. Mater. 3, 158 (2004).

[44] D. Wolf, V. Yamakov, S. R. Phillpot, A. Mukherjee, and H. Gleiter, Acta Mater. 53, 1 (2005).

[45] M. de Koning, W. Cai, and V. V. Bulatov, Phys. Rev. Lett. 91, 025503 (2003).

[46] E. Faran and D. Shilo, Phys. Rev. Lett. 104, 155501 (2010).

[47] R. Abeyarantne and S. Vedantam, J. Mech. Phys. Solids 51, 1675 (2003).

[48] V. V. Bulatov, L. L. Hsiung, M. J. Tang, A. Arsenlis, M. C. Bartelt, W. Cai, J. N. Florando, M. Hiratani, M. Rhee, G. Hommes, T. G. Pierce, and T.D. de la Rubia, Nature (London) 440, 1174 (2006).

[49] B. Devincre, T. Hoc, and L. Kubin, Science 320, 1745 (2008).

[50] T. Zhu, J. Li, A. Samanta, A. Leach, and K. Gall, Phys. Rev. Lett. 100, 025502 (2008).

[51] Y. Fan, Y. N. Osetsky, S. Yip, and B. Yildiz, Phys. Rev. Lett. 109, 135503 (2012). 